\title{
Long-range electron binding to quadrupolar molecules
}

\author{
C. Desfrançois, ${ }^{1}$ Y. Bouteiller, ${ }^{1}$ J.P. Schermann, ${ }^{1}$ \\ D. Radisic, ${ }^{2}$ S.T. Stokes, ${ }^{2}$ K.H. Bowen, ${ }^{2}$ N.I. Hammer, ${ }^{3}$ R.N. Compton ${ }^{3}$ \\ ${ }^{1}$ Laboratoire de Physique des Lasers, CNRS - Université Paris-Nord, Villetaneuse 93430, France \\ ${ }^{2}$ Chemistry Department, Johns Hopkins University, Baltimore, 21218, MD \\ ${ }^{3}$ Department of Physics, The University of Tennessee, Knoxville, Tennessee, 37996
}

An excess electron can be bound to a molecule in a very diffuse orbital as a result of the longrange contributions of the molecular electrostatic field. Following a systematic search, we report experimental evidence that such quadrupole binding occurs for the trans-succinonitrile molecule $(\mathrm{EA}=$ $20 \pm 2 \mathrm{meV}$ ), while the gauche-succinonitrile conformer supports a dipole-bound anion state $(\mathrm{EA}=108$ $\pm 10 \mathrm{meV}$ ). Theoretical calculations at the DFT/B3LYP level support these interpretations and give electron affinities of 20 and $138 \mathrm{meV}$, respectively.

PACS numbers: 3315 . Ry, 3360-q, 3460+z 
An excess electron can be trapped in the molecular potential well essentially created by the long-range attractive fields plus a short-range repulsive core potential. In the case of the dipolar potential, a minimum dipole moment of about $2.5 \mathrm{D}$ is required for the formation of stable dipolebound anions. Electron affinities (EAs) ranging from few tenths to few tens of meV have been predicted ${ }^{1}$ and measured ${ }^{2-4}$. The average distance between such a loosely bound electron and the polar molecule is then as large as several tens of $\AA$ and the excess electron mainly experiences the molecular asymptotic dipole field. When the dipole moment becomes too large, e.g. in $\mathrm{NaCl}^{-}$, the excess electron orbital is localized close to the positive end (e.g. $\left.\mathrm{Na}^{+}\right)$and is then more characteristic for a conventional valence anion state 5 .

We here reexamine the problem of the extension of dipole-bound to quadrupole-bound anions. The possibility that an electron might be bound to a molecule as a result of its quadrupole moment was first considered theoretically by Jordan and Liebman ${ }^{6}$. Several theoretical 5,7 and experimental 8 studies with conflicting results have since been devoted to this problem. On the experimental side, there has been no firm evidence for the existence of weakly bound anions due to the quadrupolar interactions alone. Experimental data, from Rydberg electron transfer to the formamide dimer ${ }^{9}$ and photoelectron spectroscopy of $\mathrm{MgO}$ cluster anions ${ }^{8}$ have been interpreted in terms of quadrupolebound anions. However, it can be argued that these interpretations might not be valid due to the lack of theoretical support in the formamide case and to rather well localized orbitals, close to $\mathrm{Mg}$ atoms, in the case of $\mathrm{MgO}$ clusters, with rather large EA values $(0.5-1 \mathrm{eV})$. In this paper we present the first solid evidence of very weak electron binding to a molecular quadrupole in the case of transsuccinonitrile $\left(\mathrm{NC}-\mathrm{CH}_{2}-\mathrm{CH}_{2}-\mathrm{CN}\right)$. This conformer has a zero dipole moment and rather large quadrupole moments resulting from the two opposite $\mathrm{C}-\mathrm{N}$ dipoles, separated by about $5 \AA$. In contrast to the formamide dimer, their positive ends point inside the molecular frame, in a region where the excess electron cannot be accommodated due to the repulsion with the closed-shell molecular orbitals. 
In that case, we have to consider the presence of a quadrupolar potential plus a polarization potential as responsible for the long-range electron binding 10,11 .

A nearly universal means for the preparation of weakly bound anions is the Rydberg Electron Transfer (RET) technique. It provides a signature of the nature of the produced anions since Rydberg level $\left(\mathrm{n}^{*}\right)$ dependencies for the formation of either atomic 12 or dipole-bound 3,13 anions are always sharply peaked, in contrast with the case of most valence anions. For these very weakly bound anion states, this RET diagnosis can be further confirmed by experiments using the field-detachment technique ${ }^{14}$. With these techniques, we have conducted a systematic search for true, very weakly bound, quadrupole-bound anions. The presence of two identical polar chemical groups in a head-to-tail configuration was an obvious choice. We have measured $n^{*}$-dependencies of anion creation rates for several molecules, either saturated or unsaturated aromatic or aliphatic and substituted by two $\mathrm{CN}, \mathrm{CO}$ or $\mathrm{NO}_{2}$ groups, and for dimers of some polar molecules. RET data for these systems either exhibit only smooth valence anion behavior or mixed smooth and peaked behavior, with no observable electric field detachment for fields up to $30 \mathrm{kV} / \mathrm{cm}$. In those cases either quadrupole anion states are too loosely bound to be observed or do not exist (1,4-cyclohexanedione, acetonitrile dimer) or they are too strongly coupled to valence states (1,4-dicyanobenzene, 1,4-dinitrobenzene, fumaronitrile) which preclude their characterization. We here report on the succinonitrile (SN) molecule which displays all properties characteristic from very weakly bound anion formation.

RET studies were made at Université Paris-Nord (UPN) ${ }^{10}$ and at the University of Tennessee (UT) ${ }^{13}$. A neutral molecular beam is created by seeding helium with succinonitrile followed by expansion through a pulsed valve. This beam crosses downstream a beam of laser-excited Rydberg atoms, $\operatorname{Xe}(n f)\left(\mathrm{n}^{*} \sim \mathrm{n}\right)$ at $\mathrm{UPN}$ or $\mathrm{Rb}(n d)\left(\mathrm{n}^{*}=\mathrm{n}-1.35\right)$ at UT. Anions are created by charge-exchange between the molecular beam and the Rydberg atomic beam and they are mass-analyzed in a time-offlight mass spectrometer. Electric field detachment measurements at UPN are performed by means of a 
set of three planar grids perpendicular to the anion path ${ }^{14}$. In the anion photoelectron spectroscopic studies performed at Johns Hopkins University (JHU), the succinonitrile anions are generated in a microplasma, mass-selected and then photodetached with an argon ion laser ${ }^{2}$. The resulting electrons are then energy-analyzed with a resolution of about $20 \mathrm{meV}$.

Let us first consider the predictions that can be inferred from the structure of the neutral parents. The succinonitrile molecule possesses a remarkable property which is the presence of two nearly energetically equivalent conformations (see Table I): a trans conformation with a null dipole moment and large quadrupole moments and a gauche conformation with a large dipole moment $\mu_{\mathrm{g}} \approx 5.7 \mathrm{D}$ and relatively small quadrupole moments 15,16 . Present $a b$ initio calculations, at the MP2/aug-cc-pVDZ level (with ZPE corrections), give an energy difference between the trans and gauche conformers of 34 meV. Assuming an internal rovibrational temperature lower than the heating temperature, the relative population of the gauche conformer can be estimated to be lower than $26 \%$, most of the molecules being in the trans conformation. In the absence of any available experimental value for the quadrupole moments, our calculations provide us the traceless values of $\mathrm{Q}_{\mathrm{xx}}=+21.6$ a.u., $\mathrm{Q}_{\mathrm{yy}}=+20.7$ a.u. and $\mathrm{Q}_{\mathrm{zz}}=$ -42.3 a.u., for the trans conformer. This corresponds to a charge distribution very close to the cylindrical symmetry with $Q=-42$ a.u., i.e. a large negative value. We can thus anticipate the existence of two different anion states corresponding to electron attachment to molecules in the trans (quadrupole-bound) and gauche (dipole-bound) conformations.

The $\mathrm{n}^{*}$-dependencies of the measured $\mathrm{SN}^{-}$anion formation rates in RET collisions are displayed in Fig. 1. The main characteristic of this curve is the sharply peaked feature around $\mathrm{n}^{*}=12$, characteristic of the creation of anions which excess electrons are very weakly bound in diffuse orbitals. However, a small plateau is seen around $\mathrm{n}^{*}=20$ before dropping almost to zero above $\mathrm{n}^{*}=28$. In studies performed at UT, for which the Rydberg excitation scheme leads to a higher sensitivity, there is also an additional very small, but reproducible, peak at low $n^{*}$ values, around $n^{*}=6-7$. From the peak values of the RET curve, and with the help of a semi-empirical law verified for a large number of 
weakly bound anions ${ }^{17}, \mathrm{EA}=23 \mathrm{eV} / \mathrm{n}^{* 2.8}$, we derive the EA-values of $115 \mathrm{meV}\left(\mathrm{n}^{*}=6.65\right)$ and 22 $\mathrm{meV}\left(\mathrm{n}^{*}=12\right)$, for the excess electron binding energies. Fitting the experimental curve with a simple curve-crossing model for RET [9] leads exactly to the same values. The bound state at $22 \mathrm{meV}$ is also independently confirmed by a field-detachment experiment for anions, obtained in RET collisions with $\mathrm{Xe}(12 \mathrm{f})$ Rydberg atoms. The corresponding detaching field values, in the vicinity of $12 \mathrm{kV} / \mathrm{cm}$, are also characteristic of the creation of non-covalent anions with excess electrons in diffuse orbitals and provide an EA value of $18 \mathrm{meV}{ }^{17}$. Signal levels are too low and the binding energy is too high to obtain field detachment curves for the $115 \mathrm{meV}$ state.

Despite this experimental evidence of the formation of weakly bound anions in RET to SN, the existence of several neutral conformations raises the question of the nature of the excess electron binding. The main peak of the RET curve is similar to those expected for dipole-bound anions in which the parent molecules possess dipole moments between 3 and $4 \mathrm{D}^{3,13}$. However, SN does not possess such a dipole moment: it is 0 for the trans conformer and $5.7 \mathrm{D}$ for the gauche conformer. This provides the first indication that the main peak cannot correspond to dipole-bound anion formation. On the other hand, the small peak at low $\mathrm{n}^{*}$ is likely to correspond to dipole-bound gauche anion formation, as also strongly suggested from photoelectron spectroscopy data obtained at JHU which give an EA of $108 \pm 10 \mathrm{meV}$ for $\mathrm{SN}$ anions produced directly in the micro-plasma at the beam exit. Previous studies with the anion photoelectron method show that weakly bound anions with EA $<30$ $\mathrm{meV}$ are difficult to observe with this technique and that anions are generally formed in their lowest configurations. It is thus likely that only the dipole-bound anions, corresponding to the high-dipole gauche conformation and to the most stable anion species, are being observed with this technique. The EA value measured by photodetachment is indeed in very good agreement with the low ${ }^{*}$ feature of the RET curve and it fits well with a binding energy of an excess electron bound to a molecular dipole of about $6 \mathrm{D}$. These data provide strong evidence for our interpretation that the observed weakly bound anions, in the main peak of the RET data, can be attributed only to the null dipole conformation of 
succinonitrile. Thus, the present observations constitute the first clear experimental demonstration of the existence of anions with excess electrons in very diffuse orbitals created from a closed-shell molecule with a null dipole and large quadrupole moments.

In order to test this interpretation, we have performed quantum chemistry and semi-empirical calculations on weakly bound anions of SN. First, density functional theory (DFT/B3LYP) calculations have been performed on neutral and anion conformers, with optimized appropriate diffuse basis sets that have been proven to give accurate dipole-bound EA-values for ware dimer anions ${ }^{18}$. It seems that this method and basis set are also appropriate for nitrile molecules since we obtain, for the dipolebound anion of acetonitrile, $\mathrm{EA}=11 \mathrm{meV}$, in fair agreement with the experimental value of $18 \mathrm{meV}$ and very close to the most accurate ab initio value of $13 \mathrm{meV}^{19}$. The results for SN are given in Table I and fit very well the present experimental data: we obtain $\mathrm{EA}=20 \mathrm{meV}$ for the trans conformer and $\mathrm{EA}=138 \mathrm{meV}$ for the gauche conformer. For the trans and gauche anion conformers, the corresponding diffuse excess electron wavefunctions (anion HOMO) are displayed in Fig. 3. It is clearly seen that the gauche conformer corresponds to a dipole-bound anion in which the excess electron is mainly located on the positive side of the total charge distribution. On the contrary, in the trans anion conformer, the excess electron orbital looks like a torus about the z-axis of the two opposite $\mathrm{CN}$ dipoles. The weakly bound electron is thus mainly located close to the plane perpendicular to this axis, which is indeed the plane of the more attractive quadrupolar potential, due to the negative quadrupole moment value.

The potential energy surfaces of the neutral and anion species along the coordinate that connect the trans and gauche conformations are represented in Fig. 4. It is clearly seen that, contrary to the neutral case, the gauche dipole-bound conformer is the most stable form of the anion. It is well separated from the trans quadrupole-bound conformer by an energy barrier $(>100 \mathrm{meV})$ at least as high as in the neutral, which prevents interconversion. 
A key question is whether it is the overall quadrupole potential that binds the electron or local dipole potentials. In the case of SN, i.e. for negative quadrupole moments, the terminal nitrogen atoms possess negative partial charges while the attached carbon atoms are more or less positively charged. An excess electron bound by one local $\mathrm{CN}$ dipole would then have to be localized close to the corresponding carbon atom, where valence interactions are at least as important as electrostatic ones. The weak binding energies measured here and the above calculations rather show that the excess electron occupies a diffuse orbital, close to the plane perpendicular to the two opposite dipoles as displayed in Fig. 3, in which it is mainly submitted to the long-range part of the quadrupolar interaction.

From the present positive result regarding the formation of stable but weakly bound anions of trans succinonitrile $(\mathrm{Q} \approx-42$ a.u. $)$ and our negative results for 1,4 -cyclohexanedione $(\mathrm{Q} \approx-37$ a.u. $)$, we provide a rough estimate of the empirical critical negative quadrupole moment value, $\mathrm{Q}_{\min } \approx-40$ a.u., required to bind an excess electron. This value is much higher than the previously reported value $(-5.2$ a.u. $\left.{ }^{20}\right)$ but it is very much in line with the values derived for positive quadrupole moments $\left(+41\right.$ a.u. $\left.{ }^{20}\right)$ and from semi-empirical electrostatic model calculations (-41 a.u. $\left.{ }^{11}\right)$.

In summary, we here present the first conclusive evidence for the existence of quadrupolebound negative ions and empirically derive a minimum value for the quadrupole moment required to form a stable anion. The succinonitrile molecule studied here is particularly interesting because it can give birth to both dipole-bound and quadrupole-bound anions, as both observed in RET experiments. Although the trans conformation of neutral succinonitrile is the neutral global minimum, the lowest lying negative ion state is the dipole-bound anion of the gauche conformation, as evidenced from photodetachment measurements on anions directly produced in a micro-plasma. When starting from neutrals with subsequent RET ionization, quadrupole-bound trans anions are mainly observed, both during the formation process (RET $\mathrm{n}^{*}$-dependencies) and few microseconds later (field-detachment 
experiments), because an energy barrier prevents their interconversion towards the dipole-bound anion state.

The authors wish to thank G. Gutsev, S. Berry, and M. Gutowski for valuable discussions on loosely bound anions. RNC and KHB each acknowledge support from the National Science Foundation under grant numbers, CHE-9981945 and CHE-0211522, respectively. 


\section{Table I}

Calculated total energies $\mathrm{E}$ for the neutral and anion conformers of succinonitrile (SN) as obtained by full optimization at the DFT/B3LYP level, using home made optimized basis sets 18 . The corresponding calculated and experimental EAs and the calculated dipole and quadrupole moment values of the neutrals are also given.

\begin{tabular}{|c|c|c|c|c|c|c|c|c|}
\hline $\begin{array}{c}\text { SN } \\
\text { conformer }\end{array}$ & $\begin{array}{c}\text { E neutral } \\
\text { (a.u.) }\end{array}$ & $\begin{array}{c}\text { E anion } \\
\text { (a.u.) }\end{array}$ & $\begin{array}{c}\text { EA calc. } \\
(\mathrm{meV})\end{array}$ & $\begin{array}{c}\text { EA exp. } \\
(\mathrm{meV})\end{array}$ & $\begin{array}{c}\mu_{\mathrm{Z}} \\
(\mathrm{D})\end{array}$ & $\begin{array}{c}\mathrm{QXX}_{\mathrm{Xx}} \\
\text { (a.u.) }\end{array}$ & $\begin{array}{c}\mathrm{Q}_{\mathrm{YY}} \\
\text { (a.u.) }\end{array}$ & $\begin{array}{c}\mathrm{Q}_{\mathrm{ZZ}} \\
\text { (a.u.) }\end{array}$ \\
\hline SN trans & 264.326996 & 264.327739 & 20 & $20 \pm 2$ & 0.00 & -44 & +23 & +21 \\
\hline SN gauche & 264.325188 & 264.330246 & 138 & $108 \pm 10$ & 5.73 & -21 & +14 & +7 \\
\hline
\end{tabular}




\section{Figure captions:}

Figure 1: Rydberg Electron Transfer (RET) curves for succinonitrile anion production as a function of the effective Rydberg quantum number $\mathrm{n}^{*}$ involved in the electron transfer collisions with either $\mathrm{Xe}(n f)$ states (full squares) or $\mathrm{Rb}(n d)$ states (open squares). The full line is a fit to these experimental points, using a curve-crossing model ${ }^{17}$ with the only parameter being the excess electron binding energy EA.

Figure 2: Anion photoelectron spectrum for succinonitrile anions produced in a supersonic ion beam source. The main peak $a$ corresponds to the transition between the anion and neutral ground states, from which $\mathrm{EA}=108 \pm 10 \mathrm{meV}$ is determined, while peaks $b$ and $c$ correspond to transitions towards excited rovibrational levels of the neutral.

Figure 3: Highest Occupied Molecular Orbital (HOMO) for the trans (left) and gauche (right) conformers of succinonitrile anions, in 3D perspective view, using the gOpenMol visualization software. Both plots correspond to the same isocontour value of 0.003 a.u..

Figure 4: Potential energy surfaces for neutral and anion succinonitrile along the dihedral angle connecting the trans and gauche conformers. Neutral points (squares) have been directly obtained from DFT/B3LYP calculations while anion points (circles) have been calculated from the neutral electrostatic parameters and a semi-empirical model ${ }^{11}$ of multipole-bound anions. The order of stability between trans and gauche conformers is reversed in the neutral and the anion but there is still a rather high-energy barrier that prevents interconversion 


\section{References}

$1 \quad$ W. Garrett, Chem.Phys.Lett. 5, 393 (1970).

2 J. V. Coe, G. H. Lee, J. G. Eaton, et al., J.Chem.Phys. 92, 3980 (1990).

3 C. Desfrançois, H. Abdoul-Carime, N. Khelifa, et al., Phys.Rev.Lett. 73, 2436 (1994).

4 M. K. Scheller, R. N. Compton, and L. S. Cederbaum, Science 270, 1160 (1995).

5 G. L. Gutsev, P. Jena, and R. J. Bartlett, J.Chem.Phys. 111, 504 (1999).

6 K. D. Jordan and J. F. Liebman, Chem.Phys.Lett. 253, 143 (1979).

$7 \quad$ M. Gutowski and P. Skurski, Chem.Phys.Lett. 303, 65 (1999).

8 M. Gutowski, P. Skurski, X. Li, et al., Phys.Rev.Lett. 85, 3145 (2000).

9 C. Desfrançois, V. Périquet, S. Carles, et al., Chem.Phys. 239, 475 (1998).

10 C. Desfrançois, H. Abdoul-Carime, and J. P. Schermann, Int.J.Modern.Phys. 10, 1339 (1996).

11 H. Abdoul-Carime and C. Desfrançois, Eur.Phys.J..D 2, 149 (1998).

12 M. Reicherts et al, Europhys.Lett. 40, 129 (1997).

13 R. N. Compton and N. I. Hammer, in Advances in Ghase-Phase Ion Chemistry, (2001), Vol. 4, p. 257.

14 C. Desfrançois, H. Abdoul-Carime, C. Adjouri, et al., Europhysics.Lett. 26, 25 (1994).

15 G. Cardini, Righini, and S. Califano, J.Chem.Phys. 95, 679 (1991).

16 O. I. Fengler, Spectrochemica Acta A 57, 1627 (2001).

17 C. Desfrançois, Phys.Rev.A 51, 3667 (1995).

18 C. Desfrançois, V. Périquet, Y. Bouteiller, et al., J.Phys.Chem. 102, 1274 (1998).

19 P. Skurski, M. Gutowski, and J. Simons, Int.J.Quantum.Chem. 80, 1024 (2000).

M. V. N. Prasad, R. F. Wallis, and R. Herman, Phys.Rev.B 40, 5924 (1989). 
Figure 1:

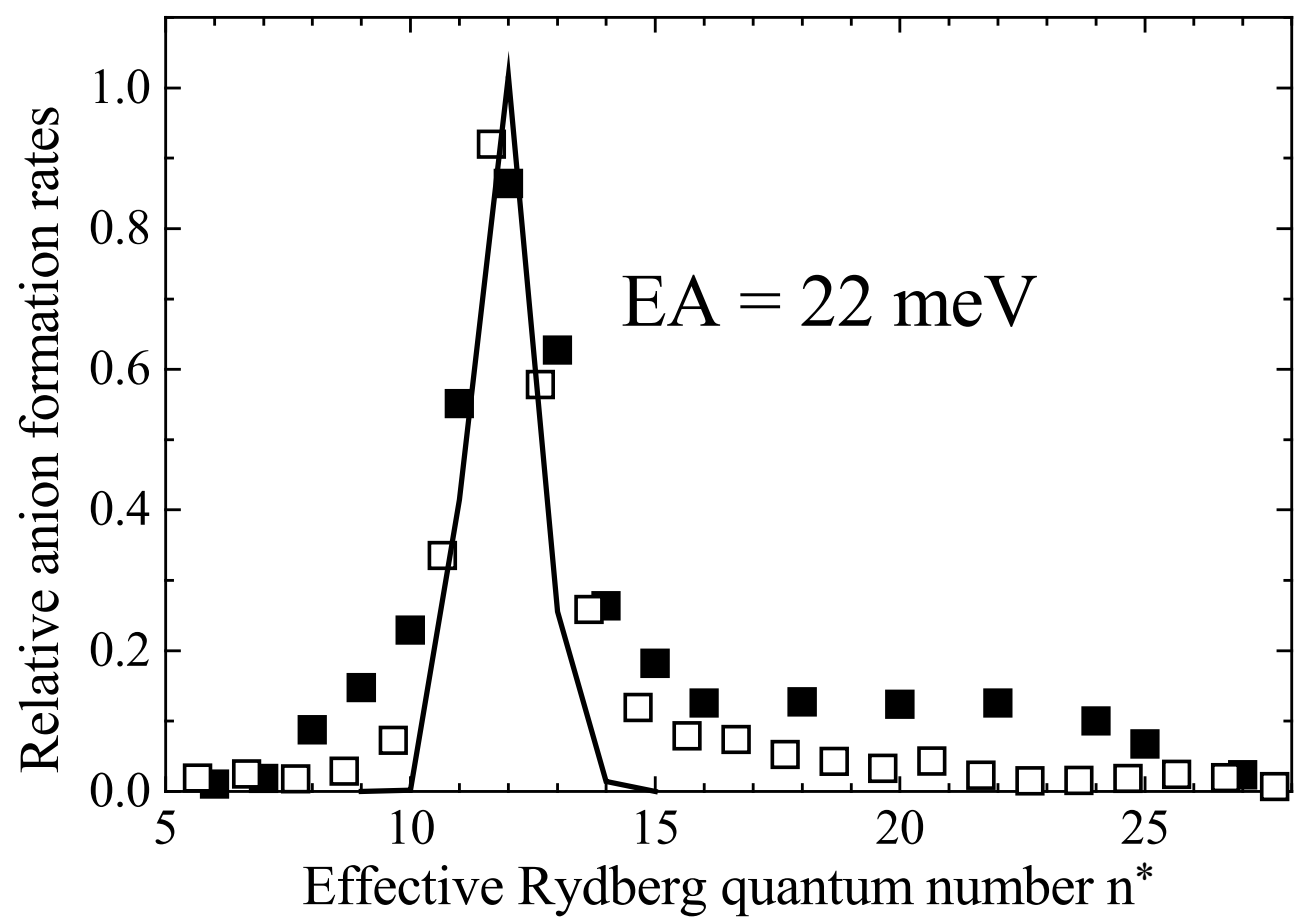

Figure 2:

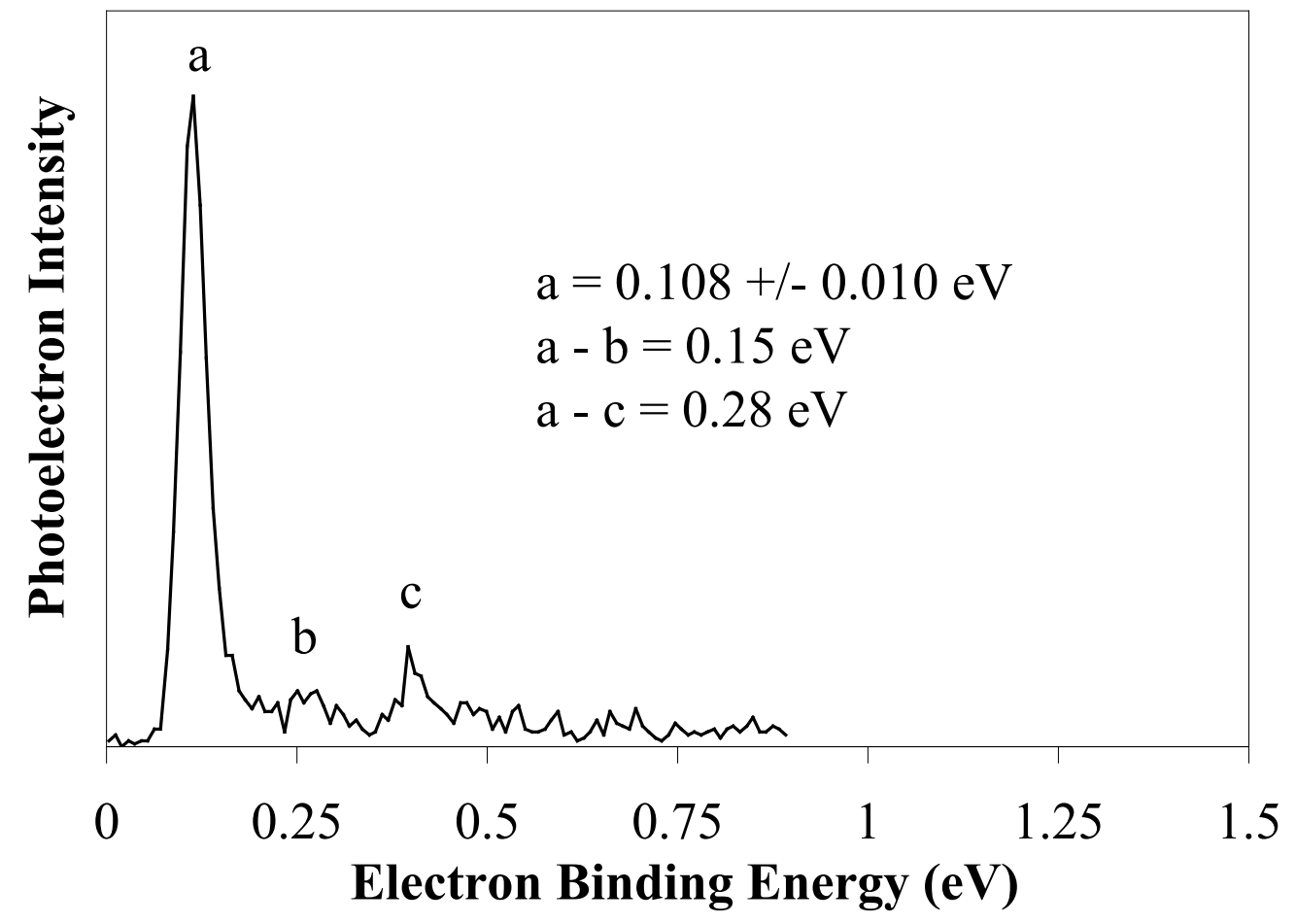


Figure 3:
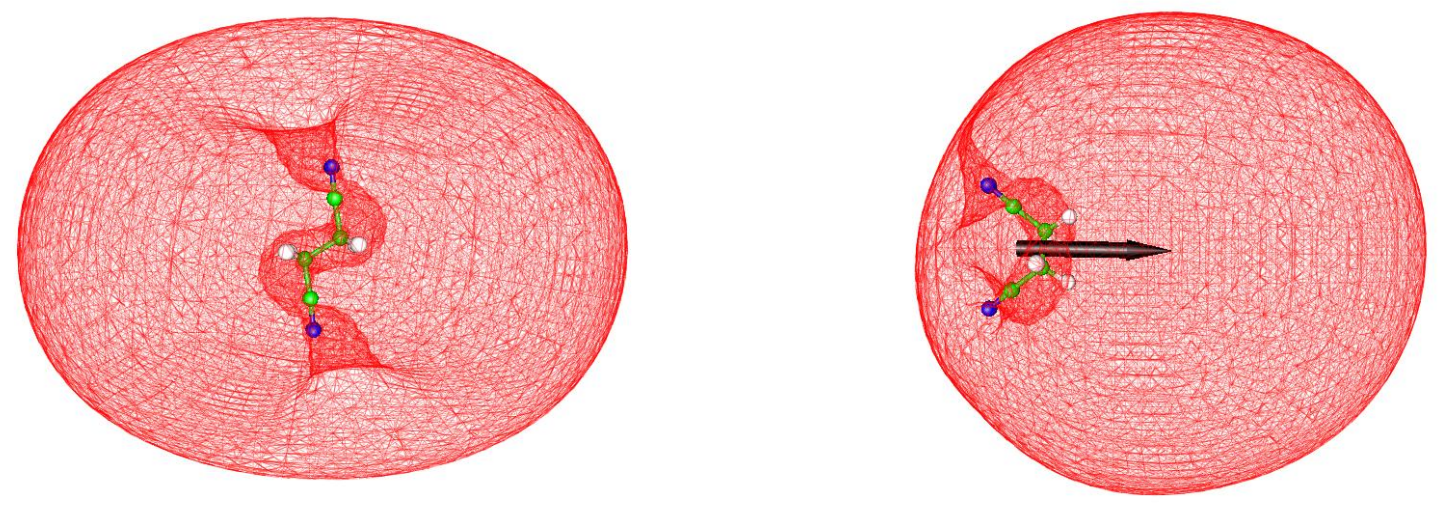

Figure 4:

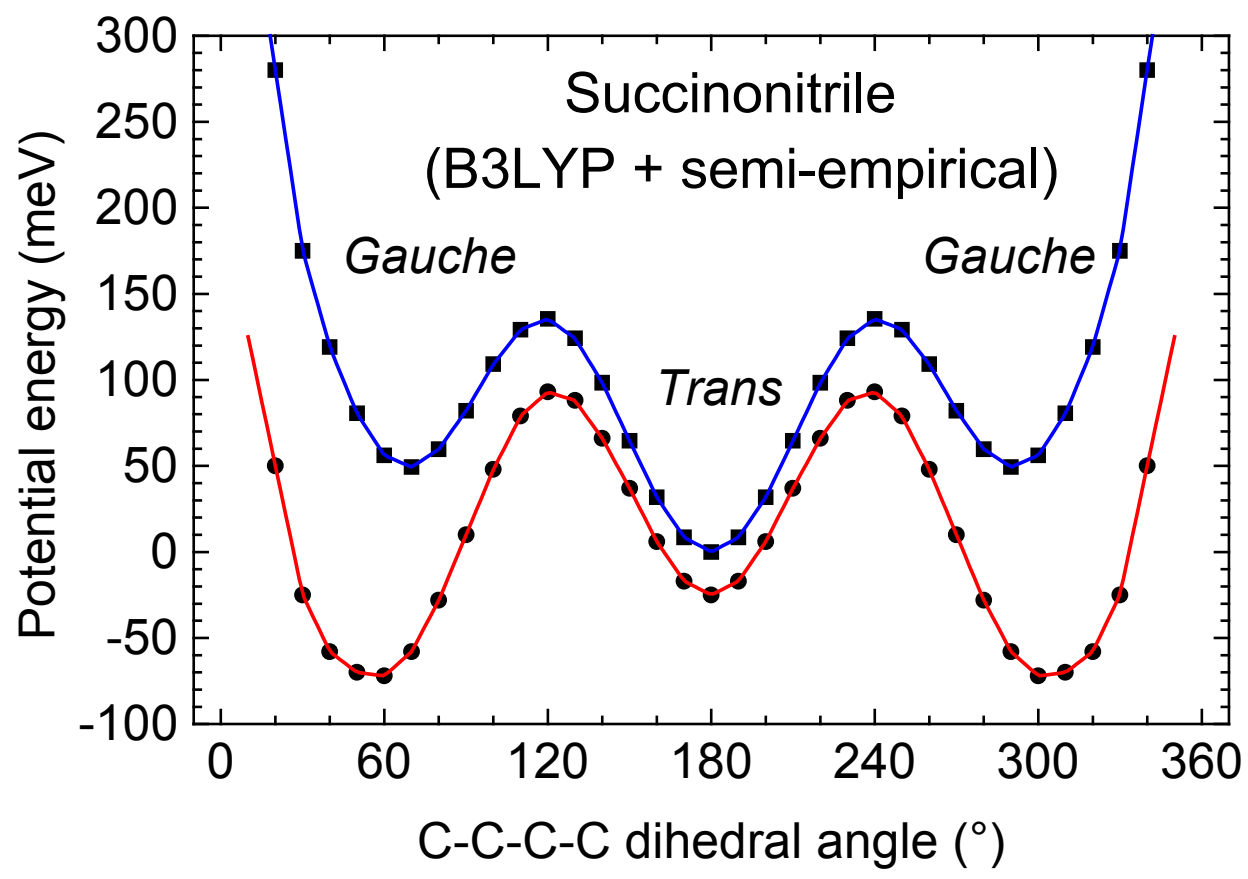

